\title{
Entanglement of coherent states and decoherence*
}

\author{
Radim Filip $^{1}$, Jaroslav Řeháček ${ }^{1}$ and Miloslav Dušek ${ }^{1,2}$ \\ ${ }^{1}$ Department of Optics, Palacký University, 17 listopadu 50, 77200 Olomouc, \\ Czech Republic \\ ${ }^{2}$ Institute of Physics, Slovak Academy of Sciences, Dúbravská cesta 9, 84228 Bratislava, \\ Slovakia
}

Received 24 May 2001, in final form 10 September 2001

Published 27 September 2001

Online at stacks.iop.org/JOptB/3/341

\begin{abstract}
The possibility of producing entangled superpositions of strong coherent states is discussed. A recent proposal by Howell and Yeazell (2000 Phys. Rev. A 62 012102) of a device that entangles two strong coherent states is modified. It is shown that the modified scheme can generate non-classical states that violate Bell inequality. Moreover, a detailed analysis of the effect of losses and decoherence on the degree of entanglement is accomplished. It reveals the high sensitivity of the device to any disturbances and the fragility of generated states.
\end{abstract}

Keywords: Entanglement, decoherence, nonlocality

\section{QND entangling device}

Recently, an interesting idea has been proposed [1] on how to entangle two strong coherent fields with a single photon (that can appear in one of two different paths) and generate a six-mode Schrödinger cat-like state utilizing non-linear Kerr interaction. The idea was inspired by recent work on a similar subject [2]. The original scheme [1] for entangling 'macroscopic' fields is outlined in figure 1 without a beamsplitter (BS) and detectors $\mathrm{D}_{1}$ and $\mathrm{D}_{2}$. The idea is as follows: first, a non-separable single-photon state

$$
\left(|1\rangle_{12}|0\rangle_{13}+i|0\rangle_{12}|1\rangle_{13}\right) / \sqrt{2}
$$

is produced using the single-photon source (SPS) and a 50:50 beamsplitter. Second, an attempt is made to transfer the whichpath uncertainty of the photon into the entanglement of strong coherent fields generated by the coherent sources (CS) by means of quantum non-demolition measurement (QND) of the number of photons in modes 12 and 13. QND measuring devices operate by the cross-Kerr interaction described by the Hamiltonian

$$
H_{\mathrm{QND}}=\hbar \pi\left(\hat{n}_{12} \hat{n}_{23}+\hat{n}_{13} \hat{n}_{33}\right),
$$

where $\hat{n}$ are photon-number operators of the corresponding modes. The strengths of the interactions (2) are carefully

* This paper is dedicated to Professor Jan Peřina on the occasion of his 65th birthday. chosen to yield the accumulated phase shift of $\pi$ in modes 23 or 33 provided a single photon is present in modes 12 or 13 , respectively. This means that the presence of a single photon, for instance, in mode 12 will cause the outputs of modes 24 and 25 to be switched completely. Working in the Schrödinger picture, the output six-mode state can be straightforwardly calculated as follows

$$
|\Phi\rangle=\frac{1}{\sqrt{2}}\left(\left|\phi_{1}\right\rangle|0\rangle_{12}|1\rangle_{13}+\left|\phi_{2}\right\rangle|1\rangle_{12}|0\rangle_{13}\right),
$$

where

$$
\begin{aligned}
& \left|\phi_{1}\right\rangle=\left|\alpha_{2}\right\rangle_{24}|0\rangle_{25}|0\rangle_{34}\left|\alpha_{3}\right\rangle_{35} \\
& \left|\phi_{2}\right\rangle=|0\rangle_{24}\left|\alpha_{2}\right\rangle_{25}\left|\alpha_{3}\right\rangle_{34}|0\rangle_{35} .
\end{aligned}
$$

Here $\alpha_{2} \neq 0$ and $\alpha_{3} \neq 0$ are complex amplitudes proportional to the amplitudes of the coherent fields fed into the inputs 21 and 31.

However, if we are interested only in the modes 34,35 and 24, 25, we cannot see any entanglement among them. It is the photon leaving the apparatus in modes 12 and 13 and carrying which-way information which destroys the entanglement among the remaining four modes. This is the weak point of the original scheme. To erase the which-way information that resides in the 12 and 13 two-mode field, we propose to superimpose these modes at a 50:50 beamsplitter as shown in figure 1 . Two single photon detectors $D_{1}$ and $D_{2}$ are attached to the outputs of the beamsplitter. After mixing the 


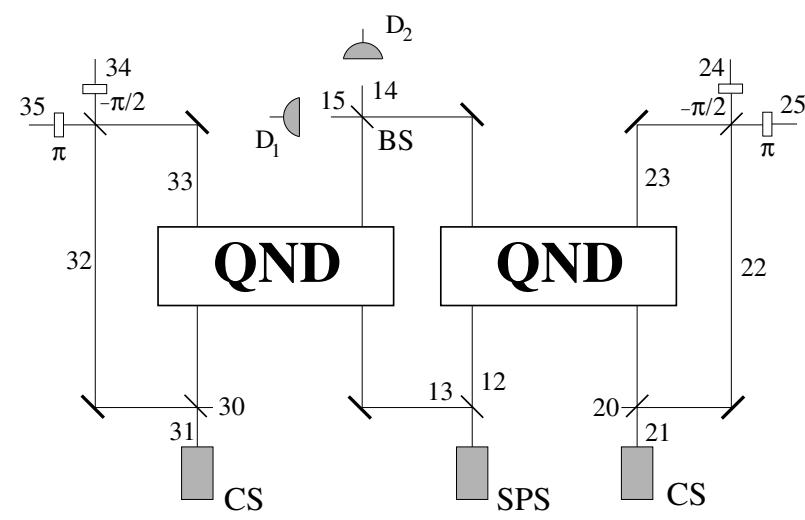

Figure 1. Entangling apparatus. QND=quantum non-demolition measuring devices; $\mathrm{CS}=$ coherent state sources; $\mathrm{SPS}=$ single-photon source; $\mathrm{BS}=$ beamsplitter; $\mathrm{D}_{1}, \mathrm{D}_{2}=$ single-photon detectors.

12 and 13 beams at the beamsplitter the state of the six-mode field reads

$$
\begin{aligned}
|\Phi\rangle= & \frac{1}{2}\left[-\left(\left|\phi_{1}\right\rangle-\left|\phi_{2}\right\rangle\right)|0\rangle_{14}|1\rangle_{15}\right. \\
& \left.+i\left(\left|\phi_{1}\right\rangle+\left|\phi_{2}\right\rangle\right)|1\rangle_{14}|0\rangle_{15}\right] .
\end{aligned}
$$

Now, depending on the result of the single-photon detection, the four-mode state of interest becomes

$$
\left|\Phi_{1}\right\rangle=N_{+}\left(\left|\phi_{1}\right\rangle+\left|\phi_{2}\right\rangle\right)
$$

if the $\mathrm{D}_{1}$ detector fires, or

$$
\left|\Phi_{2}\right\rangle=N_{-}\left(\left|\phi_{1}\right\rangle-\left|\phi_{2}\right\rangle\right)
$$

if $\mathrm{D}_{2}$ does. $N_{+}$and $N_{-}$are normalization factors ${ }^{3}$. We recall that for $\left|\alpha_{2}\right|,\left|\alpha_{3}\right| \gg 1$ the four-mode states $\left|\phi_{1}\right\rangle$ and $\left|\phi_{2}\right\rangle$ are well separated 'macroscopic' states. In such a way, a perfect source of the 'macroscopic' entangled field can be realized using a gate triggered by the $\mathrm{D}_{1}$ and $\mathrm{D}_{2}$ detectors so that only one of the states (6) and (7) is allowed to go through.

Ignoring the result of the single-photon detection one gets a mixture of states $\left|\Phi_{1}\right\rangle$ and $\left|\Phi_{2}\right\rangle$. The idea used in this report to modify the original scheme is in fact an implementation of the well known and much discussed quantum eraser [3].

Actually, the amended set-up (figure 1) is unnecessarily too complicated; it contains redundant parts. One can easily do with just one QND device. Let us remove the rightmost Mach-Zehnder (MZ) interferometer and its QND device from the set-up. The output state will change (up to the normalization) into the superposition $\left(|0\rangle_{34}\left|\alpha_{3}\right\rangle_{35}+\right.$ $\left|\alpha_{3}\right\rangle_{34}|0\rangle_{35}$ ), supposing that a click at detector $D_{1}$ has been registered. After using two additional beamsplitters in 34 and 35 paths and re-labelling the output modes, the four-mode entangled state equivalent to (6) (or (7)) is obtained again. Moreover, on the same basis even multipartite GHZ states of coherent fields can be produced by simple beamsplitting.

\section{Entanglement and nonlocality}

Before discussing decoherence, which could be a serious obstacle in realizing the 'macroscopic' entangler in the

${ }^{3} N_{ \pm}=\left[2 \pm 2 \exp \left(-2|\alpha|^{2}\right]^{(-1 / 2)}\right.$ laboratory, let us briefly discuss some interesting properties of the 'cat' state (7). First of all one may ask to which extent the state (7) is entangled. We will adopt mutual information as a convenient measure of entanglement [4]. It is defined by the difference between the sum of von Neumann entropies $S_{1}$ and $S_{2}$ of subsystems 1 and 2 and the von Neumann entropy of the composite system $S$ :

$$
I=S_{1}+S_{2}-S \text {. }
$$

Here subsystem 1 consists of modes 24 and 25; subsystem 2 consists of modes 34 and 35. Mutual information (8) is zero if the subsystems 1 and 2 are uncorrelated $\left(\rho=\rho_{1} \otimes \rho_{2}\right)$. Its maximum value still explainable by classical correlations is $I=S^{4}$.

We will consider symmetric inputs $\alpha_{2}=\alpha_{3}=\alpha \neq 0$. Notice that defining new orthogonal vectors

$$
\begin{aligned}
|\downarrow\rangle_{i} & =\frac{1}{2}\left[\left(\frac{1}{M_{+}}+\frac{1}{M_{-}}\right)|0 \alpha\rangle_{i}+\left(\frac{1}{M_{+}}-\frac{1}{M_{-}}\right)|\alpha 0\rangle_{i}\right], \\
|\uparrow\rangle_{i} & =\frac{1}{2}\left[\left(\frac{1}{M_{+}}-\frac{1}{M_{-}}\right)|0 \alpha\rangle_{i}+\left(\frac{1}{M_{+}}+\frac{1}{M_{-}}\right)|\alpha 0\rangle_{i}\right],
\end{aligned}
$$

where $i=1,2$ and $M_{ \pm}$are normalization factors ${ }^{5}$, the state (7) can be rewritten to the form resembling the spin $1 / 2$ singlet state:

$$
\left|\Phi_{2}\right\rangle=\frac{1}{\sqrt{2}}\left(|\uparrow\rangle_{1}|\downarrow\rangle_{2}-|\downarrow\rangle_{1}|\uparrow\rangle_{2}\right) .
$$

For this reason, state (7) shows properties completely analogous to the properties of the spin $1 / 2$ singlet state for any value of $\alpha \neq 0$. Namely, the mutual information between the systems 1 and 2 is maximal,

$$
I=2 \ln 2
$$

and independent of $\alpha$. This maximally violates the $2 \times 2$ Bell inequalities [6] and can be simply proven in the following way: noting that the two-dimensional subspace spanned by vectors $|\uparrow\rangle,|\downarrow\rangle$, living in the Hilbert space of the first system, is isomorphic to a spin-half particle space, one can define unitary operations

$$
\begin{aligned}
& |\uparrow\rangle_{i} \rightarrow \frac{1}{\sqrt{2}}\left(\cos \theta_{i}|\uparrow\rangle_{i}+\sin \theta_{i}|\downarrow\rangle_{i}\right) \\
& |\downarrow\rangle_{i} \rightarrow \frac{1}{\sqrt{2}}\left(-\sin \theta_{i}|\uparrow\rangle_{i}+\cos \theta_{i}|\downarrow\rangle_{i}\right)
\end{aligned}
$$

which are in a sense analogous to spin rotations. The Belltype experiment then consists of two 'rotations' according to the recipe (11) performed by two possibly space-like separated observers, followed by realistic yes-no detection performed on each spin-like system. Let us assign them values $z_{i}=-1,1$ if the states $|\downarrow\rangle$ or $|\uparrow\rangle$ are detected. Each detection has only two possible outcomes. The results $X$ and $Y$ of the local measurements (including 'rotations') performed by the first and second observer, respectively, can be expressed as

$$
X(\theta)=z_{1}(\theta), \quad Y(\theta)=z_{2}(\theta) .
$$

4 The diagonal representation of the density matrix of two correlated subsystems displaying maximum classically allowed correlations is of the form $\hat{\rho}=\sum_{i} p\left(a_{i}, b_{i}\right)\left|a_{i}\right\rangle_{1}\left|b_{i}\right\rangle_{2}\left\langle\left. b_{i}\right|_{2}\left\langle\left. a_{i}\right|_{1}\right.\right.$. Then one gets $S=S_{1}=S_{2}$.

${ }^{5} M_{ \pm}=\left[1 \pm \exp \left(-|\alpha|^{2}\right)\right]^{1 / 2}$. 
After the experiment is repeated many times and the two observers compare their notes, the following quantity can be estimated

$$
B=\left|C\left(\theta_{1}, \theta_{2}\right)+C\left(\theta_{1}, \theta_{2}^{\prime}\right)+C\left(\theta_{1}^{\prime}, \theta_{2}\right)-C\left(\theta_{1}^{\prime}, \theta_{2}^{\prime}\right)\right|,
$$

where the correlation function

$$
C\left(\theta_{1}, \theta_{2}\right) \equiv \sum_{j, k} X_{j} Y_{k} p\left(X_{j}, Y_{k} \mid \theta_{1}, \theta_{2}\right) .
$$

As with the two spin-half particles the use of (7) and (11) in (13) gives $B_{\max }=2 \sqrt{2}$, for an optimum set of angles. This result is independent of amplitude $\alpha$, similar to the mutual information. This exceeds the local realistic limit $B_{\max }^{\mathrm{cl}}=2$ [7]. Thus, from the point of view of entanglement and local realism the fourmode state (7) is equivalent to the maximally-entangled state of two spin-half particles. This is, of course, a consequence of the fact that the 'cat' state (7) is nothing else but a representation of the maximally-entangled spin state in a larger space-in the space of four harmonic oscillators.

\section{Decoherence}

So far we have assumed that the entangling device was ideal. This seems natural if fundamental aspects of thought device are discussed. However, if one seriously thought of experimental implementation of the entangling device in figure 1, such an assumption would clearly be non-realistic, and various imperfections and unavoidable losses would have to be considered.

The entangling device can be divided into two parts, the one-photon MZ interferometer and coherent-state MZ interferometers, with grossly different sensitivity to imperfections and losses. This can be illustrated in the simple case of losses modelled e.g. by the presence of an auxiliary beamsplitter in the paths of modes 23,33 , and 12 . In the latter case one can always balance the one-photon interferometer by introducing the same amount of losses in mode 13 , recovering the ideal output state (7) at the expense of a decreasing generation rate [8]. In such a case, if the photon is detected behind the interferometer, no information on its path is available and entanglement in the whole state is kept (if the losses were not balanced, one path would be more probable). In contrast, a similar compensation of losses in the coherent-state interferometers cannot help to save the entanglement. It is not difficult to see why. The fractions of the strong coherent beams reflected out of the coherent-state interferometer always carry a good deal of which-way information about the photon in the one-photon MZ interferometer, that can be extracted, e.g. by means of the phase measurement performed on them. Needless to say, in the limit of large $\alpha$, when the phase of the reflected beam becomes sharply defined, the reflected beams carry perfect which-way information about the photon propagating through the onephoton interferometer; this degrades the output state (7) to the mixed state.

Another likely cause of the loss of entanglement of the output state are decoherence effects due to the entanglement of the quantum system with environment. First, let us discuss decoherence in the one-photon Mach-Zehnder interferometer consisting of modes 12 and 13. We will assume the following simple (but rather general) model of decoherence,

$$
\begin{aligned}
|0\rangle_{12}|g\rangle_{\mathrm{env}} & \rightarrow|0\rangle_{12}\left|e_{1}\right\rangle_{\mathrm{env}}, \\
|1\rangle_{12}|g\rangle_{\mathrm{env}} & \rightarrow|1\rangle_{12}\left|e_{2}\right\rangle_{\mathrm{env}},
\end{aligned}
$$

where $|g\rangle_{\mathrm{env}},\left|e_{1}\right\rangle_{\mathrm{env}}$ and $\left|e_{2}\right\rangle_{\mathrm{env}}$ are three possibly nonorthogonal states of environment. Although explicit calculation of mutual information and Bell inequality violation is tedious, it is not difficult to obtain the results for large amplitude $\alpha \gg 1$. Denoting $a$ the overlap $a=\left|\left\langle e_{1} \mid e_{2}\right\rangle\right|$, the mutual information of the output state for large $|\alpha|$ reads

$$
\begin{aligned}
I \approx & 2 \ln 2+\frac{1+a}{2} \ln \left(\frac{1+a}{2}\right)+\frac{1-a}{2} \ln \left(\frac{1-a}{2}\right), \\
& |\alpha| \gg 1 .
\end{aligned}
$$

Similarly, the maximum of the Bell correlation function is reduced to

$$
B_{\max } \approx 2 \sqrt{1+a^{2}}, \quad|\alpha| \gg 1 .
$$

Note that $a^{2}$ equals the Ivanovics-Dieks-Peres lower bound [9] on the probability of the occurrence of an inconclusive result for error-free discrimination between the two non-orthogonal states of environment and, therefore, also between the states $|0\rangle_{12}$ and $|1\rangle_{12}$. Hence, one can say that as the amount of principally accessible which-way information about the photon in the one-photon MZ interferometer increases, the entanglement and non-classical character of the output state of the entangling machine become gradually destroyed.

Equations (15) and (16) should be compared with the corresponding formulas for an entangling machine with (balanced) losses present in the coherent state MZ interferometers. We will model the losses by placing four auxiliary beamsplitters with the same reflectivity $R$ into 22 , 23,32 and 33 paths. The resulting asymptotic entanglement is

$$
\begin{gathered}
I \approx 2 \ln 2+\frac{1+e^{-2 R|\alpha|^{2}}}{2} \ln \left[\frac{1+\exp \left(-2 R|\alpha|^{2}\right)}{2}\right] \\
+\frac{1-e^{-2 R|\alpha|^{2}}}{2} \ln \left[\frac{1-\exp \left(-2 R|\alpha|^{2}\right)}{2}\right], \\
\quad|\alpha| \gg 1, \quad R \rightarrow 0
\end{gathered}
$$

and corresponding asymptotic maximum of the Bell correlation function is given

$$
B_{\max } \approx 2 \sqrt{1+\mathrm{e}^{-4 R|\alpha|^{2}}}, \quad|\alpha| \gg 1, \quad R \rightarrow 0 .
$$

Notice that equations (17) and (18) can be obtained from equations (15) and (16) simply by substitution

$$
a^{2} \rightarrow \mathrm{e}^{-4 R|\alpha|^{2}}
$$

Again, this can be interpreted in terms of available whichway information. Now, the which-way information is gained by discriminating between (non-orthogonal) states $|\alpha \sqrt{R / 2}\rangle$ and $|-\alpha \sqrt{R / 2}\rangle$ of the beams reflected out of paths 23 and 33. The optimum error-free discrimination is done by mixing the beams with the reference coherent beams $|i \alpha \sqrt{R / 2}\rangle$ at two mixing beamsplitters [10]. The probability of the inconclusive 

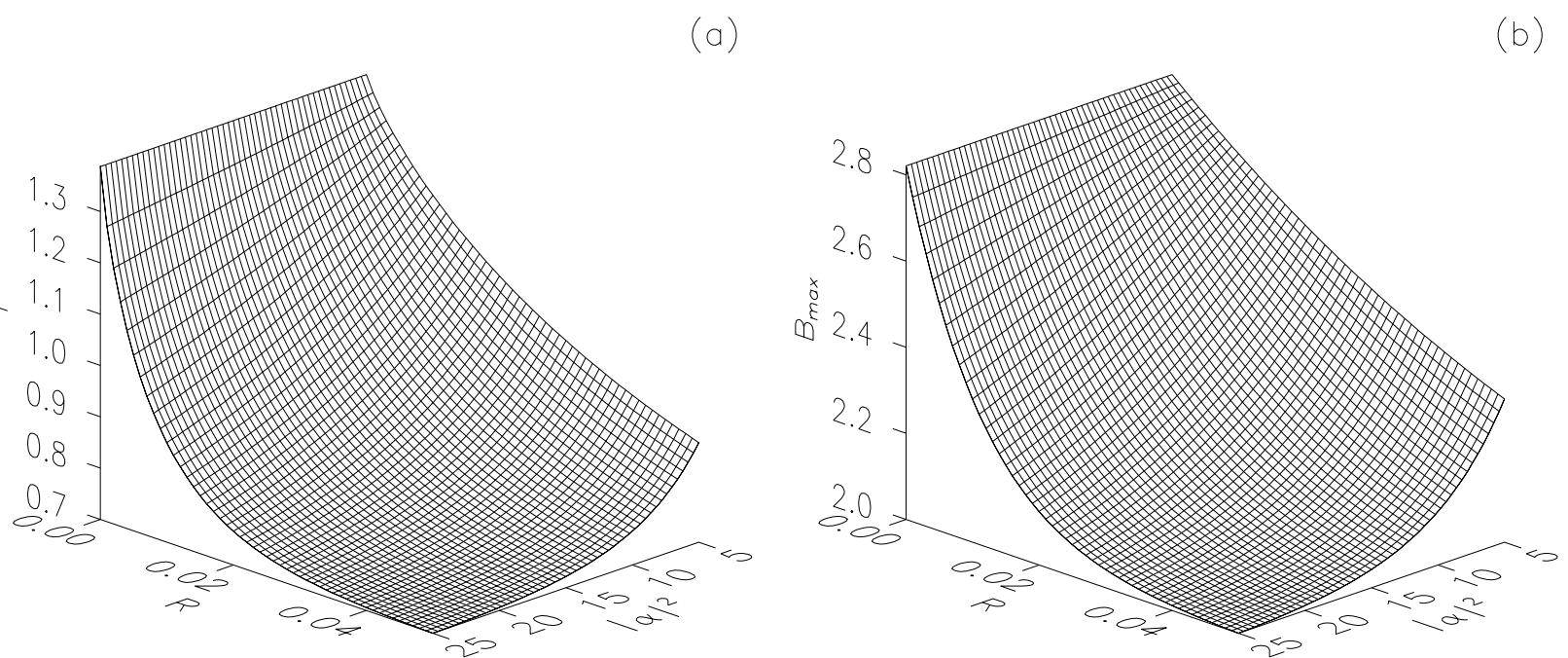

Figure 2. (a) Mutual information $I$ and $(b)$ the maximum value of the Bell correlation function $B_{\max }$ are shown for different input mean numbers of photons $|\alpha|^{2}$ and reflectivities $R$ of the beamsplitter's simulating losses.

result: no photons detected after the mixing at both outputs, is $P_{\text {inconcl. }}=\exp \left(-2 R|\alpha|^{2}\right)$ in either case. The path of the photon is revealed by getting at least one conclusive result out of the two ideal error-free measurements in the right and left part of the entangling apparatus. The probability of the unfortunate event of getting two inconclusive results is $P_{\text {inconcl. }}^{\text {total }}=P_{\text {inconcl. }}^{2}=\exp \left(-4 R|\alpha|^{2}\right)$, which is just the right-hand side of equation (19). This suggests that the loss of entanglement and non-locality caused by both effects, the decoherence in the one-photon interferometer and losses or decoherence in the coherent-state interferometers, have common information-theoretical origin.

Although equations (17)-(18) and (15)-(16) have the same functional dependence on the amount of available whichway information, their implications for the feasibility of the generation of the 'macroscopic-cat' state (7) dramatically differ. The decoherence in the one-photon MZ interferometer is not a key limiting factor for engineering such superpositions for equations (15) and (16) does not depend on the intensity of the input coherent state $|\alpha|^{2}$. In contrast to this, the relative amount of losses in the coherent-state interferometers which can be tolerated exponentially decreases with increasing input intensity, see equations (17) and (18). This precludes the entangling of arbitrarily separated coherent states.

Forgetting about macroscopical separability, the presented device can still be a useful tool for generating interesting nonclassical states. This is illustrated in figure 2. The degree of entanglement $I$ in the presence of small losses is shown in figure 2(a). Notice that for large $|\alpha|$ the entanglement of the output state is extremely sensitive to the presence of losses. With an increasing amount of losses the output state becomes a mixed state and the correlations between output modes become explainable classically. However, it can be seen that the generation of output states having non-classical correlations is possible for small input intensities. A similar discussion also holds for the maximum attainable value of the Bell correlation function $B_{\max }$, see figure $2(b)$. Here part of the figure significantly exceeding the local-realistic limit $B=2$ in a small area adjacent to plane $R=0$ indicates the range of parameters for which the generation and detection of nonclassical states is possible.

It follows that the experimental generation of non-classical states using the proposed device is possible for small intensities $|\alpha|^{2}$. Such states are interesting from the point of view of possible experiments on quantum nonlocality, or experiments utilizing quantum entanglement, but they are far from being entangled 'macroscopic' states.

\section{Conclusion}

We have shown that the 'entangling' apparatus proposed in [1] can be modified to produce entangled pairs of coherent states, which can be used to test nonlocality. Our device also uses a Kerr nonlinear medium which helps to extend the one-photon non-separable superposition to the four-mode entangled superposition of strong coherent fields. The new important point is a post-selection based on interferometric measurement on the one-photon subsystem. This erases which-way information that has prevented the creation of the desired entangled state. We have proved that the states prepared by our prescription can violate Bell-like inequalities. We have also studied to what extent losses and decoherence can degrade the produced state. This is important with respect to potential experimental realization. Unfortunately, the preparation procedure is very sensitive to decoherence and especially to losses in the strong-field interferometers. However, a set of realistic values in parameter space still exists for which entropy of states exceeds the classical level and even Bell inequality can be violated.

\section{Acknowledgment}

This research was supported under the project LN00A015 of the Ministry of Education of the Czech Republic and the European Union project EQUIP (contract IST-1999-11053). 


\section{References}

[1] Howell J C and Yeazell J A 2000 Phys. Rev. A 62012102

[2] Sanders B C and Milburn G J 1989 Phys. Rev. A 39694

Sanders B C 1992 Phys. Rev. A 456811

Sanders B C 1992 Phys. Rev. A 462966

Wielinga B and Sanders B C 1993 J. Mod. Opt. 40192

Sanders B C, Lee K S and Kim M S 1995 Phys. Rev. A 52735

Rice D A and Sanders B C 1998 Quantum. Semiclass. Opt. 10 L41

Sanders B C and Rice D A 1999 Opt. Quantum Electron. 31 525

Sanders B C and Rice D A Phys. Rev. A 61013805

Rice D A, Jaeger G and Sanders B C 2000 Phys. Rev. A 62 012101

[3] Hillery M and Scully M O 1983 Quantum Optics, Experimental Gravitation, and Measurement Theory ed P Meystre and M O Scully (New York, Plenum) p 65 Scully M O, Englert B G and Walther H 1991 Nature 351111 Kwiat P G, Steinberg A M and Chiao R Y 1992 Phys. Rev. A 457729
Kwiat P G, Steinberg M and Chiao R Y 1994 Phys. Rev. A 49 61

[4] Barnett S M and Phoenix S J D 1989 Phys. Rev. A 402404

[5] Mann A, Sanders B C and Munro W J 1995 Phys. Rev. A 51 989

Fu H, Wang X and Solomon A I 2000 Preprint arXiv:quant-ph/0105099

[6] Einstein A, Podolsky B and Rosen N 1935 Phys. Rev. 47777 Bohm D 1952 Phys. Rev. 85166

Bohr N 1935 Nature 13665

[7] Bell J S 1964 Physics 1195

Clauser J F, Horne M A, Shimony A and Holt R A 1969 Phys. Rev. Lett. 23880

[8] Hendrych M, Dušek M and Haderka O 1996 Acta Physica Slovaka 46393

[9] Ivanovic I D 1987 Phys. Lett. A 123257

Dieks D 1998 Phys. Lett. A 126303

Peres A 1988 Phys. Lett. A 12819

[10] Huttner B, Imoto N, Gisin N and Mor T 1995 Phys. Rev. A 51 1863

Phillips L S, Barnett S M, and Pegg D T 1998 Phys. Rev. A 58 3259 